\title{
PHYSICAL AND MECHANICAL ANALYSIS OF THE RECOVERY OF GRANITE PÉTREOS WASTE AS ADDED GRAFTING IN PORTLAND CEMENT CONCRETE
}

\author{
Fernando Gabriel do Nascimento Júnior ${ }^{1}$, Thalia Rabelo Ferreira ${ }^{2}$, Arlindo Rubens de Oliveira \\ Frota $^{3}$, Murilo Ferreira dos Santos ${ }^{4}$, David Barbosa de Alencar ${ }^{5}$, Darlei dos Anjos Lavor ${ }^{6}$
}

\author{
1236 Centro Universitário do Norte (UNINORTE). Manaus-AM. \\ ${ }^{4}$ Instituto de Ensino Superior Blauro Cardoso de Mattos (FASERRA). Manaus-AM. \\ ${ }^{5}$ Instituto de Tecnologia e Educação Galileo da Amazônia (ITEGAM). Manaus-AM.
}

Email: fernandofla13@gmail.com; thaliarabelo@hotmail.com.br; arof_@hotmail.com; mullsantos@gmail.com; david002870@hotmail.com; darleilavor27@gmail.com

Received: April 10"th 2019

Accepted: April 29th, 2019

Published: June 30th, 2019

Copyright @2016 by authors and Institute of Technology Galileo of Amazon (ITEGAM). This work is licensed under the Creative Commons Attribution International License (CC BY 4.0). https://creativecommons.org/lice nses/by/4.0/

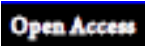

\begin{abstract}
The increase in waste generated by the construction industry (RCC) in recent years, without adequate disposal, has accumulated concerns not only to government agencies but also to the environment and public health in Brazil, which varies from region to region. region. The use of these RCCs incorporated in concrete to reduce these clusters has been a solution increasingly employed by companies in this segment that are concerned with the future of our. Therefore, this study presents a feasibility in the use of marbled residues as a large aggregate in total replacement of crushed stone No. 0 in conventional concrete. For this, laboratory experiments were carried out with the objective of characterizing all the materials used. After the characterization, the axial compressive strengths of the hardened and cured Test Bodies were verified in the ages of 7 and 28 days, divided in two groups with $4 \mathrm{CPs}$ each. The average of the results of the residue with a value of $25.02 \mathrm{MPa}$ and the conventional concrete with gravel, of $27.32 \mathrm{MPa}$, makes it possible to use the residue for small buildings such as: sidewalks, gutters and small non-structural slabs bearing.
\end{abstract}

Keywords: Concrete. Granite residue. Use.

\section{ANÁLISE FÍSICA E MECÂNICA DO REAPROVEITAMENTO DOS RESÍDUOS PÉTREOS DE GRANITO COMO AGREGADO GRAÚDO EM CONCRETO DE CIMENTO PORTLAND.}

\begin{abstract}
RESUMO
O aumento dos resíduos gerados pela construção civil (RCC) nos últimos anos, sem uma destinação adequada, tem acumulado preocupações não só aos órgãos governamentais mais também ao que se refere a meio ambiente e a saúde pública no Brasil, do qual varia de região para região. A utilização desses RCC's incorporados ao concreto para a redução desses aglomerados, tem sido uma solução cada vez mais empregada por empresas desse segmento que se preocupam com o futuro do nosso planeta. Então, este estudo apresenta uma viabilidade na utilização dos resíduos de marmoraria como agregado graúdo em substituição total da brita $\mathrm{n}^{\circ} 0$ no concreto convencional. Para isso, foram realizados experimentos laboratoriais com o objetivo de caracterizar todos os materiais utilizados. Após a caracterização foram verificadas as resistências à compressão axial dos Corpos de Prova endurecidos e curados nas idades de 7 e 28 dias, divididos em dois grupos com 4 CP's cada um. A média dos resultados do resíduo com o valor de 25,02 MPa e do concreto convencional com brita, no valor de 27,32 MPa, torna-se possível a utilização do resíduo para pequenas edificações como: calçadas, sarjetas e lajes não estruturais de pequeno porte.
\end{abstract}

Palavras-Chaves: Concreto. Resíduo de Granito. Aproveitamento. 


\section{INTRODUÇÃO}

A preocupação com a quantidade de Resíduos Sólidos Urbanos (RSU) produzidos, assim como a sua destinação final, tornou-se essencial para quaisquer tipos de industrias geradoras de inúmeros segmentos.

A gestão e a disposição inadequada dos resíduos sólidos causam impactos socioambientais, tais como degradação do solo, comprometimento dos corpos d'água e mananciais, intensificação de enchentes, contribuição para a poluição do ar e proliferação de vetores de importância sanitária nos centros urbanos e catação em condições insalubres nas ruas e nas áreas de disposição final [1].

A maioria dos processos produtivos, atualmente, é fonte geradora de resíduos que se apresentam na forma de gases, líquidos ou sólidos. A produção destes rejeitos é muito maior que a capacidade de absorção da natureza, causando grande degradação ambiental, além de diversos impactos econômicos e sociais [2].

Portanto, a produção excessiva de resíduos sólidos e o uso insustentável dos recursos naturais se configuram numa lógica destrutiva e num risco para a sustentabilidade do planeta, cuja reversão depende da modificação das atitudes e práticas individuais e coletivas [3].

A indústria das rochas ornamentais gera uma enorme quantidade de efluentes. Os rejeitos em sua grande maioria são descartados em lagoas de decantação e aterros e são formados por materiais de elevada finura a partir do processo de recorte, polimento e lustro de peças, confeccionadas a partir das chapas de mármore e granito [4].

Os resíduos gerados no beneficiamento de rochas ornamentais são basicamente de dois tipos: frações de pedras e lama em forma de polpa, que se caracteriza como sendo a massa mineral resultante dos processos de serragem, polimento e corte [5].

Esta lama residual é um rejeito que se tornou um problema ambiental pela quantidade produzida. Uma forma de contribuir para o reaproveitamento desses resíduos é a utilização em concretos, pois uma boa parte dos resíduos gerados é reaproveitada e utilizada sob a forma de um novo produto, em benefício da sociedade [4].

Quanto ao uso de agregados gerados de resíduos de rochas ornamentais e para revestimento na construção civil ou na fabricação de concreto, ainda há pouco material publicado no Brasil. Alguns trabalhos encontrados na literatura evidenciam pesquisas com os resíduos da serragem de granitos e mármores, conhecidos como "lama", como adição para produção de concreto [6] na mistura com solo para aterros na produção de concreto auto-adensável [7] e o uso dos finos de serragem, polimento e lustro de rochas para revestimento na fabricação de cimento [8].

Pensando nisso, esta pesquisa foi desenvolvida com o objetivo de utilizar esses resíduos como forma de melhorar o desempenho físico e mecânico da determinação de um concreto com a resistência mecânica de $25 \mathrm{MPa}$.
A utilização desse material trará, também, uma melhor condição ao meio ambiente assim como a destinação final adequada, desses resíduos, para as marmorarias.

\section{MATERIAIS E MÉTODOS}

\section{II.1 COLETA, SELEÇÃO E PREPARAÇÃO DO RESÍDUO DE GRANITO.}

O material utilizado na pesquisa é proveniente do descarte de uma marmoraria situada na cidade de Manaus, coletado in loco, lavado e secado ao sol por três dias para retirar $100 \%$ da umidade. Para que chegássemos às características parecidas com as da brita $\mathrm{n}^{\circ} 0$ e obter uma comparação mais justa, a quebra do material foi totalmente artesanal com o auxílio de martelo e/ ou talhadeira.

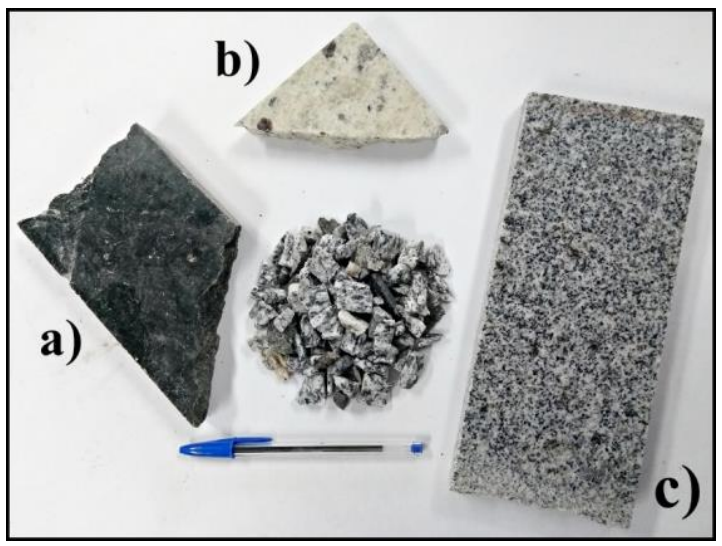

Figura 1 - Resíduos selecionados e separados de acordo com a nomenclatura. a) Granito Preto São Gabriel; b) Granito

Branco Marfim; c) Granito Cinza Corumbá. Fonte: Autores (2018).

\section{II.2 MATERIAIS UTILIZADOS PARA A COMPOSIÇÃO DOS CONCRETOS.}

\section{II.2.1 CIMENTO.}

O cimento utilizado nesta pesquisa foi do tipo CP IV-32 Super Forte da marca MIZU. A marca é uma das mais utilizadas nas edificações na região norte e mostrou ser o material mais adequado para esta pesquisa.

Para analisar as características do cimento selecionado foram utilizados os seguintes procedimentos:

Determinação da massa específica [9];

Determinação da pasta de consistência normal [10];

Determinação dos tempos de pega [11];

\section{II.2.2 AGREGADOS, MIÚDO E GRAÚDO.}

Os procedimentos utilizados para os testes de qualidade e caracterização dos agregados miúdo e graúdo foram fundamentadas de acordo com as especificações da norma [12]. Tanto a areia quanto a brita foram doações de professores do Centro Universitário do Norte (UNINORTE).

A seguir tem-se a relação das normas utilizadas para a caracterização dos materiais. 
> Determinação da composição granulométrica [13];

> Determinação da massa unitária e do volume de vazios [14];

Determinação da massa específica e massa específica aparente [15];

$>$ Determinação da massa específica, massa específica aparente e absorção de água [16].

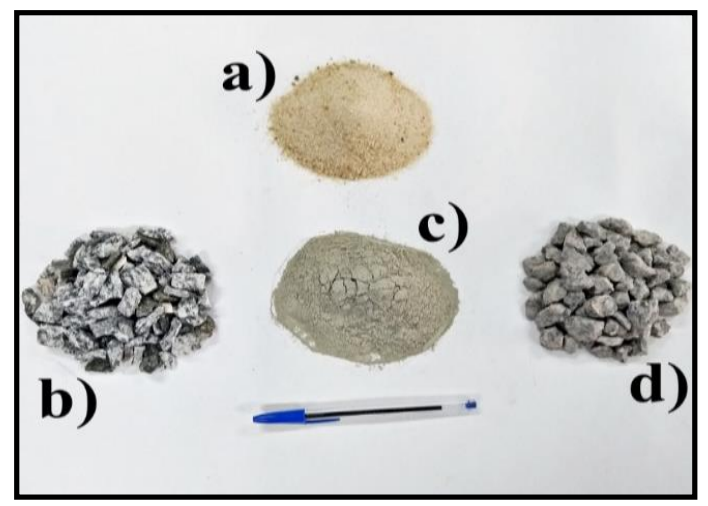

Figura 2 - Materiais expostos, agregados utilizados para a confecção do concreto. a) Areia; b) Resíduo de granito; c) Cimento Portland; d) Brita $\mathrm{n}^{\circ} 0$.

Fonte: Autores (2018).

\section{II.2.3 ÁGUA.}

A água utilizada para a confecção dos concretos para a moldagem dos corpos de prova, foi a água destilada disponibilizada no laboratório de Materiais de Construção da Instituição (UNINORTE). Para o processo de cura, a água usada para abastecer o tanque e deixar os corpos de prova submersos é a proveniente do abastecimento público da cidade de Manaus, ÁGUAS DE MANAUS S/A, também obtida pelo Laboratório de Materiais de Construção da Instituição supracitada.

\section{II.3 MOLDAGEM, CURA E RUPTURA DOS CORPOS DE PROVA.}

O processo de moldagem dos corpos de prova de concreto em estado fresco atende as especificações estabelecidas pela norma [17]. Todos os CP's, após o tempo de endurecimento de $24 \mathrm{~h}$, foram desformados e levados para o tanque de cura onde permaneceram conforme as idades estabelecidas de 7 e 28 dias. As dimensões das formas e dos CP's tem de $200 \mathrm{~mm}$ de altura e $100 \mathrm{~mm}$ de diâmetro conforme mostra a Figura 3.

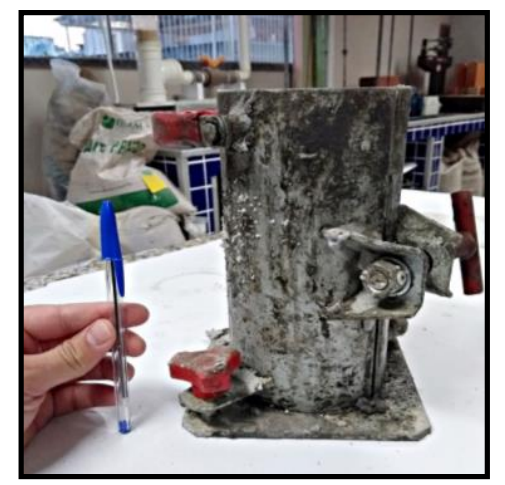

Figura 3 - Forma utilizada para a confecção dos corpos de prova.

Fonte: Autores (2018)
As rupturas foram realizadas com o auxílio da prensa hidráulica elétrica (I3025-B) da marca CONTENCO, atendendo as especificações normativas [18].

\section{II.4 ATIVIDADES REALIZADAS.}

Abaixo, na figura 4, o fluxograma mostra algumas das atividades realizadas da caracterização dos materiais para a confecção dos concretos, de forma resumida para melhor visualização.

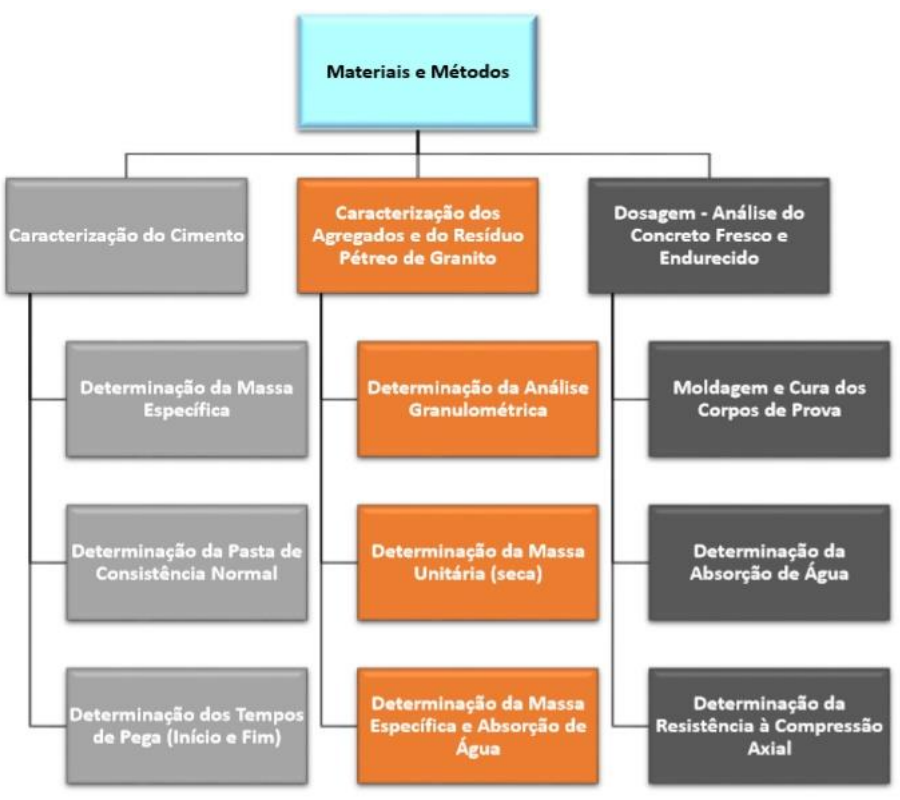

Figura 4 - Fluxograma das atividades realizadas para confecção desta pesquisa.

Fonte: Autores (2018).

\section{RESULTADOS E DISCUSSÕES}

\section{III.1 ELABORAÇÃO DO TRAÇO UNITÁRIO DOS CONCRETOS.}

De acordo com os dados coletados das análises de caracterização dos materiais, o traço experimental de referência para esta pesquisa proveio conforme a tabela 1, abaixo. A orientação empregada nesta pesquisa para a elaboração deste traço foi fundamentada através das instruções da Associação Brasileira de Cimento Portland - ABCP [19].

Tabela 1 - Traço unitário do concreto experimental de referência.

\begin{tabular}{|c|c|c|c|c|}
\hline TRAÇO & $\begin{array}{c}\text { FCK } \\
(\mathbf{M p a})\end{array}$ & SD & $\begin{array}{c}\text { FC28 } \\
(\mathbf{M p a})\end{array}$ & $\begin{array}{c}\text { FATOR } \\
\text { 31MPa (A/C) } \\
\text { TAB. }\end{array}$ \\
\hline $\mathbf{1 : 1 , 6 8 : 2 , 1 3 : 0 , 5 0}$ & 25 & 4,0 & 31,6 & 0,50 \\
\hline
\end{tabular}

\section{III.2 ANÁLISE DA ABSORÇÃO DE ÁGUA DOS CORPOS DE PROVA DE CONCRETOS.}

Após a desforma dos traços confeccionados, período de 24h, os corpos de prova foram levados para a balança de precisão e medidos seus pesos. Com o término do prazo de cura, novamente os corpos de prova foram levados a balança de precisão. A tabela 2, abaixo, demonstra essas medidas de acordo com cada traço de concreto estabelecido, sendo: seus pesos após 
desforma, seus pesos após o término do prazo de cura, o peso de absorção de água assim como a porcentagem de absorção e a média aritmética de cada $\mathrm{CP}$.

Tabela 2 - Ensaio de absorção de água dos CP's confeccionado.

\begin{tabular}{|c|c|c|c|c|c|c|}
\hline \multicolumn{7}{|c|}{ ENSAIO DE ABSORÇÃO DE ÁGUA } \\
\hline & Traços & $\begin{array}{c}\text { Massa } \\
\text { Seca } \\
(\mathrm{g})\end{array}$ & $\begin{array}{c}\text { Massa } \\
\text { Úmida } \\
(\mathrm{g})\end{array}$ & $\begin{array}{c}\text { Absorção } \\
(\mathrm{g})\end{array}$ & $\begin{array}{c}\text { Teor de } \\
\text { Absorção } \\
(\%)\end{array}$ & 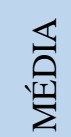 \\
\hline \multirow{2}{*}{$\begin{array}{l}\tilde{a} \\
0 \\
0\end{array}$} & $\begin{array}{c}\text { CP1 - } \\
\text { BRITA }\end{array}$ & 3724,0 & 3755,8 & 31,8 & 0,85 & \multirow{2}{*}{1,05} \\
\hline & $\begin{array}{c}\text { CP2 - } \\
\text { BRITA }\end{array}$ & 3713,4 & 3759,9 & 46,5 & 1,25 & \\
\hline \multirow{2}{*}{ 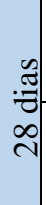 } & $\begin{array}{c}\text { CP3 - } \\
\text { BRITA }\end{array}$ & 3715,8 & 3762,2 & 46,4 & 1,25 & \multirow{2}{*}{1,19} \\
\hline & $\begin{array}{c}\text { CP4 - } \\
\text { BRITA }\end{array}$ & 3719,4 & 3761,6 & 42,2 & 1,13 & \\
\hline \multirow{2}{*}{$\begin{array}{l}: 0 \\
0 \\
0\end{array}$} & $\begin{array}{c}\text { CP1 - } \\
\text { GRANIT } \\
\text { O }\end{array}$ & 3617,7 & 3651,1 & 33,4 & 0,92 & \multirow{2}{*}{0,89} \\
\hline & $\begin{array}{c}\text { CP2 - } \\
\text { GRANIT } \\
\text { O }\end{array}$ & 3638,0 & 3669,4 & 31,4 & 0,86 & \\
\hline \multirow{2}{*}{ 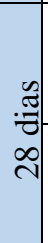 } & $\begin{array}{c}\text { CP3 - } \\
\text { GRANIT } \\
\text { O }\end{array}$ & 3633,6 & 3671,7 & 38,1 & 1,05 & \multirow{2}{*}{1,07} \\
\hline & $\begin{array}{c}\text { CP4 - } \\
\text { GRANIT } \\
\text { O }\end{array}$ & 3619,8 & 3659,3 & 39,5 & 1,09 & \\
\hline
\end{tabular}

Fonte: Autores (2018).

A seguir, a figura 5 apresenta o gráfico conforme a tabela 2 (acima).

Nota-se que os corpos de prova de concreto moldados com os resíduos pétreos de granito têm o teor de absorção de água menor do que os corpos de prova moldados com a brita $\mathrm{n}^{\circ} 0$.

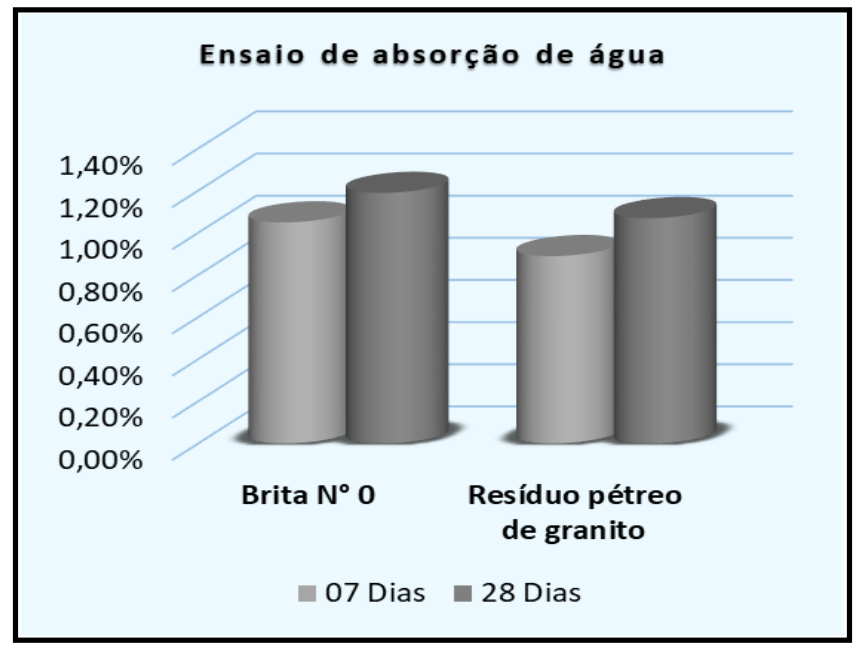

Figura 5 - Gráfico do ensaio de absorção de água dos corpos de prova dos traços experimentais.

Fonte: Autores (2018).

\section{III.3 DETERMINAÇÃO DA RESISTÊNCIA À COMPRESSÃO AXIAL DOS CORPOS DE PROVA DOS CONCRETOS ENDURECIDOS.}

A tabela 3, abaixo, exibe a ruptura dos CP's, de acordo com cada traço unitário.

Tabela 3 - Determinação da resistência mecânica dos corpos de prova de concreto.

\begin{tabular}{|c|c|c|}
\hline \multicolumn{3}{|c|}{ Resistência à compressão axial } \\
\hline \multirow{2}{*}{ TRAÇO } & \multicolumn{2}{|c|}{ Carga de ruptura (Kgf) } \\
\hline & 07 DIAS & 28 DIAS \\
\hline \multirow{2}{*}{ BRITA } & 20890,0 & 21240,0 \\
\hline & 20950,0 & 22750,0 \\
\hline \multirow{2}{*}{ GRANITO } & 18100,0 & 19780,0 \\
\hline & 19000,0 & 21730,0 \\
\hline \multicolumn{3}{|c|}{ Resistência à compressão (MPa) } \\
\hline \multirow{2}{*}{ BRITA } & 26,60 & 27,04 \\
\hline & 26,67 & 28,97 \\
\hline \multirow{2}{*}{ GRANITO } & 23,05 & 25,18 \\
\hline & 24,19 & 27,67 \\
\hline \multicolumn{3}{|c|}{ Média entre as amostras (MPa) } \\
\hline BRITA & 26,64 & 28,01 \\
\hline GRANITO & 23,62 & 26,43 \\
\hline
\end{tabular}

Ainda na tabela 3 , observa-se que existem os resultados dados pela prensa hidráulica e os resultados em MPa, assim como a média aritmética entre eles. Para melhor visualização desses resultados, a figura 6 demonstra essas representatividades através do gráfico, a seguir.

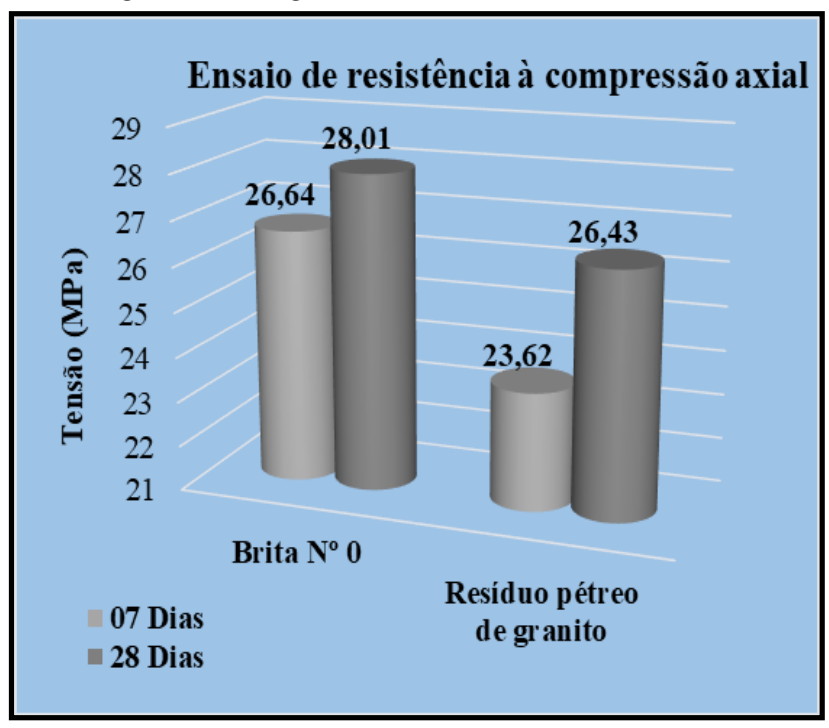

Figura 6 - Gráfico de representatividade do resultado da ruptura dos corpos de prova em MPa.

Fonte: Autores (2018).

\section{CONCLUSÃO}

Caracterizar os materiais para determinar a confecção de um outro material durável e com um bom desempenho físico e mecânico não é uma tarefa fácil. A escolha dos materiais certos nem sempre caem dos céus, requer uma tarefa árdua e persistente até que se encontrem os materiais adequados. Pensando nisso, esta pesquisa caracterizou materiais determinando tamanhos, espessuras e atendendo procedimentos para que se 
confeccionasse um concreto com resíduos pétreos de granito descartados, muitas das vezes em locais inadequados, das marmorarias. Todas as análises realizadas atendem as normas técnicas e/ ou procedimentos que foram insistentemente estudados como esta pesquisa. A análise de ruptura dos concretos dos quais todos foram moldados com uma perspectiva de Fck maior que ou igual $(\geq)$ a $25,0 \mathrm{MPa}$ atendem as expectativas tanto do traço de concreto de referência (brita $\mathrm{N}^{\circ} 0$ ) com a média de 27,32 MPa, quanto do traço de concreto com os resíduos pétreos de granito, na média igual a 25,02 $\mathrm{MPa}$. A viabilidade desse tipo de concreto em relação ao desempenho físico e mecânico pode ser melhorada uma vez que a instituição não dispõe de todos os equipamentos e aferição em dia. Então, afirma-se que os resíduos pétreos de granito descartados das marmorarias são viáveis para a utilização dos seus respectivos fins (calçadas, sarjetas ou lajes não-estrutural, uma vez que a sua resistência mecânica atende as expectativas do traço confeccionado.

\section{REFERÊNCIAS BIBLIOGRÁFICAS}

[1] BESEN, G. R. et al. Resíduos sólidos: vulnerabilidades e perspectivas. In: SALDIVA P. et al. Meio ambiente e saúde: o desafio das metrópoles. São Paulo: Ex Libris, 2010.

[2] GOME, K.; BACARJI, E. Avaliação do módulo de deformação e da durabilidade de concretos produzidos com o resíduo de beneficiamento de mármore e granito (RBMG) em substituição ao agregado miúdo. BE2008 - Encontro Nacional Betão Estrutural 2008. Disponível em: <http://www.hms.civil.uminho.pt/events/be2008/155.pdf $>$. Acesso: dezembro, 2018.

[3] BECK, U. Risk society. London: Sage Publications, 1994. GUIVANT, J. A trajetória das análises de risco: da periferia ao centro da Teoria Social. Revista Brasileira de Informação Bibliográfi ca em Ciências Sociais, Rio de Janeiro, Relume Dumará/Anpocs, n. 46, p. 3-38, 1998.

FERREIRA, L. Idéias para uma sociologia da questão ambiental no Brasil. São Paulo: Annablume, 2006.

[4] DEGEN, M. K.; VIEIRA, G. L.; CALMON, J. L.; ULIANA, J. G.; BASTOS, R. S. Concretos produzidos com resíduos provenientes do beneficiamento de rochas ornamentais como substituto parcial de cimento. ANAIS DO 55 CONGRESSO BRASILEIRO DO CONCRETO - CBC2013 - 55CBC. Disponível em:< https://www.researchgate.net/profile/Maxwell_Degen2/publicat ion/319330265_Concretos_produzidos_com residuos_provenie ntes do beneficiamento de rochas ornamentais como substit uto_parcial_de cimento/links/59a56099a6fdcc9fe95f1ef8/Conc retos-produzidos-com-residuos-provenientes-do-

beneficiamento-de-rochas-ornamentais-como-substitutoparcial-de-cimento.pdf>. Acesso: dezembro, 2018.

[5] Lima, R. C. O., Neves, G. A., carvalho, J. B. Q. Durabilidade de tijolos de solo-cimento produzidos com resíduo de corte de granito. Revista Eletrônica de Materiais e Processos, Paraíba, v. 5.2, p. 24-31, 2010.

[6] GONSALVES, J. P.; MOURA, Washington Almeida.; Dal Molin, Denise Carpena Coitinho. (2002) Avaliação da Influência da utilização do resíduo de corte de granito (RCG), como adição em propriedades mecânicas do concreto. In: IX ENCONTRO
NACIONAL DE TECNOLOGIA DO MEIO AMBIENTE CONSTRUİDO, 2002, Rio Grande do Sul. Anais... Rio Grande do Sul: ANTAC, v.2, n.1, p.53-68.

[7] LISBOA, E.M. (2004) Obtenção do concreto auto-adensável utilizando resíduo do beneficiamento do mármore e granito e estudo de propriedades mecânicas. 144f. Dissertação (Mestrado em Engenharia Civil) - Programa de Mestrado em Engenharia Ambiental, Universidade Federal de Alagoas, Alagoas.

[8] FRASCÁ, M. H. B. O. Estudo para o aproveitamento de resíduos pétreos de marmorarias, como agregados para concreto de cimento Portland. Exacta, São Paulo, v. 6, n. 1, p. 83-92, jan./jun. 2008 . Disponível em: <https://www.redalyc.org/html/810/81011705010/> Acesso: novembro, 2018.

[9] ASSOCIAÇÃO BRASILEIRA DE NORMAS TÉCNICAS. NBR 16605 - Cimento Portland e outros materiais em pó Determinação da massa específica. Rio de Janeiro. 2017.

[10] ASSOCIAÇÃO BRASILEIRA DE NORMAS TÉCNICAS. NBR 16606 - Determinação da pasta de consistência normal. Rio de Janeiro. 2017.

[11] ASSOCIAÇÃO BRASILEIRA DE NORMAS TÉCNICAS. NBR 16607 - Cimento Portland - Determinação do tempo de pega. Rio de Janeiro. 2017.

[12] NBR 7211/2005 e 7211/2009 - Agregados para concreto Especificação.

[13 ASSOCIAÇÃO BRASILEIRA DE NORMAS TÉCNICAS. NBR NM 248 - Agregados - Determinação da composição granulométrica. Rio de Janeiro. 2003.

[14] ASSOCIAÇÃO BRASILEIRA DE NORMAS TÉCNICAS. NBR NM 45 - Agregados - Determinação da massa unitária e do volume de vazios. Rio de Janeiro. 2006.

[15] ASSOCIAÇÃO BRASILEIRA DE NORMAS TÉCNICAS. NBR NM 52 - Agregado miúdo - Determinação da massa específica e massa específica aparente. Rio de Janeiro. 2009.

[16] ASSOCIAÇÃO BRASILEIRA DE NORMAS TÉCNICAS. NBR NM 53 - Agregado graúdo - Determinação da massa específica, massa específica aparente e absorção de água. Rio de Janeiro. 2009.

[17] ASSOCIAÇÃO BRASILEIRA DE NORMAS TÉCNICAS. NBR 5738 - Concreto - Procedimento para moldagem e cura de corpos de prova. Rio de Janeiro. 2015.

[18] ASSOCIAÇÃO BRASILEIRA DE NORMAS TÉCNICAS. NBR 5739 - Concreto - Ensaios de compressão de corpos-deprova cilíndricos. Rio de Janeiro. 2007.

[19] ASSOCIAÇÃO BRASILEIRA DE CIMENTO PORTLAND. Propriedades e dosagem do concreto. Módulo 2. Rio de Janeiro. 2002. 